Quim. Nova, Vol. 27, No. 1, 22-26, 2004

\title{
DETERMINAÇÃO DE CORANTES ARTIFICIAIS POR CROMATOGRAFIA LÍQUIDA DE ALTA EFICIÊNCIA (CLAE) EM PÓ PARA GELATINA
}

\author{
Marcelo Alexandre Prado e Helena Teixeira Godoy* \\ Departamento de Ciência de Alimentos, Faculdade de Engenharia de Alimentos, Universidade Estadual de Campinas, \\ CP 6121, 13083-970 Campinas - SP
}

Recebido em 31/10/02; aceito em 13/6/03

\begin{abstract}
DETERMINATION OF SYNTHETIC DYES BY HIGH PERFORMANCE LIQUID CHROMATOGRAPHY (HPLC) IN JELLY POWDER. Synthetic dyes were determined by high performance liquid chromatography in 76 samples of regular jelly and diet jelly powders, of several flavors produced by seven different manufacturers. Three sample of each product, from different batches, were analyzed. The same qualitative composition was observed for products of the same flavor from different manufacturers, but the quantitative composition varied markedly from one manufacturer to the other. There were no or few significant differences between batches from a given manufacturer, demonstrating good quality control in the use of these additives by the food industry. None of the samples exceeded the limit stipulated by Brazilian legislation.
\end{abstract}

Keywords: jelly; dyes; HPLC.

\section{INTRODUÇÃO}

O emprego de aditivos químicos em alimentos é motivo de muita polêmic, gerando controvérsias que envolvem consumidores, indústria, pesquisadores e governo. Os corantes artificiais são aditivos alimentares e têm sido objeto de muitas críticas, já que o emprego destes em alguns alimentos se justifica apenas por questões de hábitos alimentares ${ }^{1,2}$.

A cor é associada a muitos aspectos de nossa vida, influenciando nossas decisões, incluindo as que envolvem os alimentos. A aparência, segurança, aceitabilidade e características sensoriais dos alimentos são todas afetadas pela cor. Embora esses efeitos sejam associações inerentes às características psicológicas, eles interferem na escolha dos produtos ${ }^{3}$.

Muitos alimentos industrializados não apresentam cor originalmente e, em outros, a cor natural é alterada ou destruída durante o processamento e/ou estocagem, com isso o uso de corantes para suplementar ou realçar a coloração perdida e, principalmente, para aumentar a aceitabilidade do produto frente ao consumidor é um recurso muito utilizado. A idéia de consumo desses produtos dá-se primeiramente pela visão; alimentos coloridos, vistosos, são muito mais atraentes para o consumidor, e essa cor deve-se ao uso de corantes, aditivos que não são totalmente inofensivos.

Os corantes orgânicos sintéticos artificiais foram, progressivamente, substituindo os corantes naturais, devido à sua maior estabilidade, poder de coloração, maior faixa de coloração e menor preço, além de garantir a uniformidade dos alimentos produzidos em larga escala ${ }^{4}$.

Com a globalização e o conseqüente aumento das importações/ exportações, fazem-se cada vez mais necessárias a utilização de métodos mais confiáveis, eficientes e rápidos para a detecção, identificação e quantificação dos corantes. Com as restrições das legislações em relação às quantidades permitidas e as tendências futuras de se aumentar essas restrições, o problema passou a ser além da iden-

*e-mail: helena@fea.unicamp.br tificação, a quantificação desses corantes em alimentos. O Brasil permite os seguintes corantes artificiais: Tartrazina, Amarelo Crepúsculo, Amaranto, Ponceau 4R, Vermelho 40, Eritrosina, Azul Indigotina e Azul Brilhante, Verde Rápido, Azul Patente V e Azorrubina ${ }^{5-7}$.

A determinação de corantes sintéticos em alimentos tem sido feita por vários métodos espectrofotométricos ${ }^{8}$ e cromatográficos, e mais recentemente utilizando a cromatografia líquida de alta eficiên$\mathrm{cia}^{9-19}$. Prado e Godoy ${ }^{1}$ desenvolveram e validaram uma metodologia, por CLAE que, além das vantagens de eficiência na separação, rapidez e simplicidade, permite a separação e quantificação dos oito corantes permitidos em uma única análise, utilizando sistema de eluição isocrática sem a adição de par-iônico à fase móvel.

Este trabalho teve como objetivo a determinação dos corantes em pós para gelatina utilizando a CLAE. A escolha desse alimento para as análises deve-se ao fato de ser um produto ampla e habitualmente consumido, principalmente pela população infantil, e por ser um produto onde os corantes artificiais são tradicionalmente empregados em sua composição.

\section{PARTE EXPERIMENTAL}

\section{Material}

Foram analisados vários sabores de cinco marcas de gelatina comum e quatro marcas de gelatinas "diet". Para cada marca/sabor de gelatina comum, foram analisados três lotes diferentes, identificados pela data de fabricação apresentada na embalagem. As amostras de gelatina comum e "diet" foram preparadas homogeneizandose todo o conteúdo de duas embalagens em moinho de facas, com peneira de 60 mash. As análises foram sempre realizadas em duplicata. Todos os produtos foram comprados em supermercados da região de Campinas, São Paulo e Limeira, totalizando 76 amostras.

\section{Reagentes}

Os padrões de corantes artificiais foram adquiridos através da Brastóquio Imp. Ltda. O metanol utilizado com grau cromatográfico (ominsolv), a solução amoniacal e o acetato de amônio foram adqui- 
ridos da Merck Brasil e cartuchos de sep-pak $\mathrm{C}_{18}$. A água utilizada no preparo das fases móveis e das amostras foi purificada no sistema Milli-Q (Millipore). As fases móveis foram sempre filtradas em filtros Floupore (Millipore Hawp 0013) de 0,5 $\mu \mathrm{m}$ de diâmetro de poro e degaseificadas em banho ultra-sônico.

\section{Equipamentos}

Após a extração as amostras foram centrifugadas em centrífuga marca Hitachi modelo Himac CR21.

Para análise em CLAE foi utilizado cromatógrafo a líquido HP série 1050, com sistema de bombeamento isocrático e válvula injetora tipo "Rheodyne" e uma alça de amostragem de $20 \mu \mathrm{L}$ de capacidade. Um detector de arranjo de diodos (DAD) HP série 1050, acoplado a um "software HP Chemstation", que apresenta o recurso de visualização, em até cinco diferentes comprimentos de onda em uma mesma corrida cromatográfica, possibilitou a análise de todos os corantes de forma simultânea. Para esse trabalho, utilizaram-se os mesmos comprimentos de onda propostos por Prado e Godoy ${ }^{1}$, $595 \mathrm{~nm}$ para os corantes azuis, $525 \mathrm{~nm}$ para os vermelhos e $450 \mathrm{~nm}$ para os amarelos. Foi utilizada como coluna analítica a "Spherisorb" ODS-2 de 150 x 4,6 mm d.i., 5 m (Sigma-aldrich, USA), protegida por uma coluna de guarda "Micropore" 30 x 4,6 mm d.i., $\mathrm{C}_{18,}, 10 \mu \mathrm{m}$ (Varian, USA).

\section{Métodos}

Para a extração dos corantes, pesou-se entre 4 a $5 \mathrm{~g}$ de amostra, previamente homogeneizada. Devido à simplicidade da matriz as amostras foram simplesmente dissolvidas em água quente $\left(40\right.$ a $\left.50{ }^{\circ} \mathrm{C}\right)$ e o volume ajustado com água em balão volumétrico de $50 \mathrm{~mL}$. Após o ajuste de volume, cerca de $10 \mathrm{~mL}$ foram coletados e centrifugados por $10 \mathrm{~min}$ a uma rotação de $15.000 \mathrm{rpm}$. O sobrenadante foi filtrado em membrana fluoropore, HAWP 001300 (Millipore), com poros de $0,5 \mu \mathrm{m}$ e depois injetado no cromatógrafo.

\section{Etapa cromatográfica}

Antes da injeção da amostra , a coluna era condicionada, por um período inicial de $12,5 \mathrm{~min}$, a uma vazão de $0,5 \mathrm{~mL} / \mathrm{min}$, pela passagem de uma solução de água/metanol (70:30) adicionada de 0,08 mol/L de acetato de amônio. Imediatamente, a fase móvel era trocada por uma nova fase móvel constituída apenas de água/metanol, nas mesmas proporções, mas sem a presença do tampão. Com esta fase móvel os corantes são separados através de um sistema de eluição isocrática, com uma vazão de $0,5 \mathrm{~mL} / \mathrm{min}$. O tempo necessário para a separação dos oito corantes foi de $20 \mathrm{~min}$. A coluna foi condicionada, novamente, pela passagem da fase móvel tamponada por um período de 12,5 min antes de ser acionada uma nova corrida.

\section{Detecção e identificação}

Os compostos foram detectados através do detector de arranjo de diodos (DAD) na região do visível. O recurso oferecido pelo "software" permitiu o registro gráfico de cada cromatograma nos três diferentes comprimentos de onda estabelecidos: $595 \mathrm{~nm}$ para os corantes azuis, para os vermelhos a $525 \mathrm{~nm}$ e os amarelos a $450 \mathrm{~nm}$.

Os corantes foram identificados através da comparação dos tempos de retenção obtidos com soluções padrão analisadas nas mesmas condições e pelos espectros de absorção obtidos pelo DAD. A adição de padrões às amostras também foi usado como parâmetro de identificação (co-cromatografia). A quantificação foi realizada por padronização externa, construindo curvas com 5 níveis de concentração, sendo cada ponto representado pela média de três determinações ${ }^{20}$.

\section{RESULTADOS E DISCUSSÃO}

A simplicidade das matrizes permitiu que as amostras fossem apenas dissolvidas em água quente, centrifugadas, filtradas e injetadas no cromatógrafo. A etapa de centrifugação é suficiente para facilitar no processo de filtração subseqüente.

A utilização de sistema isocrático com fase móvel tamponada, apresentou grande vantagem, principalmente pela não utilização do par-iônico e pelo pequeno tempo de condicionamento necessário para o sistema cromatográfico, apenas $12,5 \mathrm{~min}$. O uso de uma fase móvel tamponada no condicionamento da coluna foi significativo, pois a presença do tampão melhorou a resolução entre os corantes tartrazina, amaranto, azul de indigotina, ponceau $4 \mathrm{R}$ e amarelo crepúsculo.

Tais dados coincidem com os encontrados em sistemas isocráticos, mudando-se o comprimento de onda ou a fase móvel dos sistemas para a obtenção de misturas de padrões de corantes artificiais $^{19}$. A fase móvel tamponada pode afetar de duas maneiras a afinidade do composto para com a fase estacionária: o tampão pode ser utilizado para suprir a ionização; e/ou para reduzir a solubilidade do corante pela fase móvel ${ }^{18}$. Esses dados confirmaram a eficiência de eluentes tamponados na separação dos corantes artificiais ${ }^{16,18}$.

Em testes preliminares, a utilização de brometo de cetiltrimetilamônio (cetrimida) necessitou de um período equivalente a dois dias para condicionar todo o sistema ${ }^{1}$. Embora a técnica de par-iônico seja a mais recomendada para a análise de corantes artificiais ${ }^{9,16,21}$, o uso desses compostos apresenta sempre o inconveniente de longos períodos de condicionamento.

As composições qualitativa e quantitativa dos corantes artificiais presentes nas amostras são apresentadas nas Tabelas 1 e 2. Dos oitos corantes permitidos pela legislação brasileira, foram encontrados nas amostra analisadas apenas a tartrazina, o amarelo crepúsculo, o amaranto, o azul de indigotina e o ponceau 4R. O amaranto foi o corante artificial quantitativamente mais utilizado pelos fabricantes de gelatina (Figura 1), e ainda esteve presente em um maior número de amostras analisadas. $\mathrm{O}$ ponceau $4 \mathrm{R}$ foi encontrado em apenas duas marcas de gelatina "diet" que, coincidentemente, só fabricam alimentos "diet". Nas outras marcas de gelatina "diet", que também fabricam pó para gelatina comum, a composição nos dois tipos de produtos foi a mesma para os diferente sabores.

As análises estatísticas foram realizadas nas amostras de gelatina com a intenção de se verificar diferenças significativas na composição quantitativa de corantes para um mesmo sabor entre as diferentes marcas, Tabelas 3 e 4 . Na maioria das amostras analisadas, ocorreu diferença significativa, nível de 5\% de significância, nas quantidades de corantes artificiais utilizadas nos mesmos sabores de diferentes marcas, chegando em algumas situações a se utilizar uma quantidade seis vezes maior de um determinado corante. Já no caso das gelatinas "diet", praticamente, não houve diferença significativa dos corantes utilizados entre os diferentes fabricantes.

Três lotes diferentes, de três fabricantes diferentes, de pó para gelatina comum foram analisados. Nas Tabelas 3 e 4, estão apresentadas as concentrações para cada lote das diferentes marcas, divididos por sabores. Pelo resultado da análise de variância verifica-se que a maioria dos fabricantes manteve um bom controle na utilização desses aditivos.

A Figura 2 mostra os cromatogramas de duas amostras de gelatina, uma normal e outra "diet", nos três comprimentos de onda. 
Tabela 1. Teores de corantes artificiais $(\mathrm{mg} / 100 \mathrm{~mL})$ em pó para gelatina comum*

\begin{tabular}{|c|c|c|c|c|c|c|}
\hline \multirow[t]{2}{*}{ Sabor } & \multirow[t]{2}{*}{ Corante } & \multicolumn{5}{|c|}{ Marca } \\
\hline & & A & B & $\mathrm{C}$ & $\mathrm{D}$ & $\mathrm{E}$ \\
\hline \multirow[t]{3}{*}{ Morango } & tartrazina & & & & & $0,8 \pm 0,1$ \\
\hline & amaranto & $4,2 \pm 0,2 \mathrm{a}$ & $3,7 \pm 0,3 b$ & $1,62 \pm 0,02 c$ & $1,40 \pm 0,06 \mathrm{c}$ & $4,14 \pm 0,07 \mathrm{a}$ \\
\hline & amarelo crep. & $3,4 \pm 0,2 \mathrm{a}$ & $2,7 \pm 0,0 b$ & $3,5 \pm 0,5 \mathrm{a}$ & $2,25 \pm 0,04 b$ & $1,6 \pm 0,2 \mathrm{c}$ \\
\hline \multirow[t]{2}{*}{ Abacaxi } & tartrazina & $1,57 \pm 0,01 \mathrm{a}$ & $2,3 \pm 0,3 b$ & $3,3 \pm 0,1 \mathrm{c}$ & $2,9 \pm 0,2 \mathrm{c}$ & $0,8 \pm 0,2 \mathrm{~d}$ \\
\hline & amarelo crep. & $0,53 \pm 0,02 \mathrm{a}$ & $0,46 \pm 0,04 a$ & $3,3 \pm 0,5 b$ & $0,20 \pm 0,04 c$ & $0,12 \pm 0,06 \mathrm{c}$ \\
\hline Cereja & amaranto & $10,6 \pm 0,3 \mathrm{a}$ & $6,6 \pm 0,5 b$ & & $4,79 \pm 0,01 \mathrm{c}$ & $3,0 \pm 0,2 \mathrm{~d}$ \\
\hline \multirow[t]{2}{*}{ Limão } & tartrazina & $1,61 \pm 0,04 a$ & $1,56 \pm 0,05 \mathrm{a}$ & $2,05 \pm 0,00 \mathrm{~b}$ & $1,97 \pm 0,02 b$ & \\
\hline & indigotina & $0,35 \pm 0,00 \mathrm{a}$ & $0,60 \pm 0,02 b$ & $0,67 \pm 0,03 b$ & $0,20 \pm 0,01 \mathrm{c}$ & \\
\hline \multirow[t]{2}{*}{ Uva } & amaranto & $3,2 \pm 0,4 \mathrm{a}$ & $3,10 \pm 0,01 \mathrm{a}$ & $3,46 \pm 0,07 a$ & $2,49 \pm 0,07 b$ & \\
\hline & indigotina & $0,64 \pm 0,08 \mathrm{a}$ & $0,49 \pm 0,05 b$ & $0,46 \pm 0,09 b$ & $0,22 \pm 0,03 c$ & \\
\hline \multirow[t]{3}{*}{ Framboesa } & tartrazina & & & & & $0,9 \pm 0,2$ \\
\hline & amaranto & $3,0 \pm 0,2 \mathrm{a}$ & & & $2,48 \pm 0,03 b$ & $4,1 \pm 0,2 b$ \\
\hline & amarelo crep. & $1,03 \pm 0,01 \mathrm{a}$ & & & $1,5 \pm 0,1 b$ & \\
\hline \multirow[t]{2}{*}{ Tangerina } & tartrazina & $0,50 \pm 0,02 \mathrm{a}$ & & & $0,17 \pm 0,00 \mathrm{~b}$ & \\
\hline & amarelo crep. & $1,1 \pm 0,2 \mathrm{a}$ & & & $0,5 \pm 0,3 b$ & \\
\hline \multirow[t]{2}{*}{ Pêssego } & tartrazina & $0,43 \pm 0,07 \mathrm{a}$ & & & & $1,05 \pm 0,09 \mathrm{~b}$ \\
\hline & amarelo crep. & $0,55 \pm 0,02 \mathrm{a}$ & & & & $0,8 \pm 0,4 \mathrm{a}$ \\
\hline Tutti- & amaranto & $1,31 \pm 0,07 \mathrm{a}$ & & & & $4,2 \pm 0,3 b$ \\
\hline \multirow[t]{2}{*}{ Frutti } & tartrazina & $1,08 \pm 0,02 \mathrm{a}$ & & & & $2,93 \pm 0,01 \mathrm{~b}$ \\
\hline & amarelo crep. & $2,13 \pm 0,05 a$ & & & & $0,85 \pm 0,07 \mathrm{~b}$ \\
\hline
\end{tabular}

* médias e estimativa do desvio padrão $(n=4)$; letras iguais na mesma linha indicam que não há diferença significativa ao nível de $5 \%$ de significância

Tabela 2. Teores de corantes artificiais $(\mathrm{mg} / 100 \mathrm{~mL})$ em pó para gelatina “diet”*

\begin{tabular}{|c|c|c|c|c|c|}
\hline \multirow[t]{2}{*}{ Sabor } & \multirow[t]{2}{*}{ Corante } & \multicolumn{4}{|c|}{ Marca } \\
\hline & & A & $\mathrm{D}$ & $\mathrm{F}$ & G \\
\hline \multirow[t]{2}{*}{ Morango } & amaranto & $5,66 \pm 0,08 \mathrm{a}$ & $2,0 \pm 0,9 \mathrm{~b}$ & & $3,72 \pm 0,0 \mathrm{c}$ \\
\hline & amarelo crep. & $4,16 \pm 0,09 a$ & $0,77 \pm 0,05 b$ & & \\
\hline Abacaxi & tartrazina & & $2,13 \pm 0,04 \mathrm{a}$ & $1,92 \pm 0,08 \mathrm{a}$ & $2,0 \pm 0,2 \mathrm{a}$ \\
\hline \multirow[t]{2}{*}{ Cereja } & amaranto & $10,7 \pm 0,4 a$ & $4,13 \pm 1,3 b$ & $2,36 \pm 0,01 \mathrm{c}$ & $4,6 \pm 0,7 b$ \\
\hline & ponceau 4R & & & $6,8 \pm 1,1 \mathrm{a}$ & $5,6 \pm 0,7 \mathrm{a}$ \\
\hline \multirow[t]{2}{*}{ Limão } & tartrazina & $1,82 \pm 0,02 \mathrm{a}$ & & $3,8 \pm 0,1 b$ & \\
\hline & indigotina & $0,54 \pm 0,06 a$ & & $1,1 \pm 0,3 b$ & \\
\hline \multirow[t]{2}{*}{ Framboesa } & amaranto & $4,2 \pm 0,4 \mathrm{a}$ & & $3,2 \pm 1,2 \mathrm{a}$ & $4,34 \pm 0,02 \mathrm{a}$ \\
\hline & amarelo crep. & $5,8 \pm 0,1 \mathrm{a}$ & & $6,5 \pm 1,9 a$ & \\
\hline
\end{tabular}

*médias e estimativa de desvio padrão $(\mathrm{n}=4)$; letras iguais na mesma linha indicam que não há diferença significativa ao nível de 5\% de significância
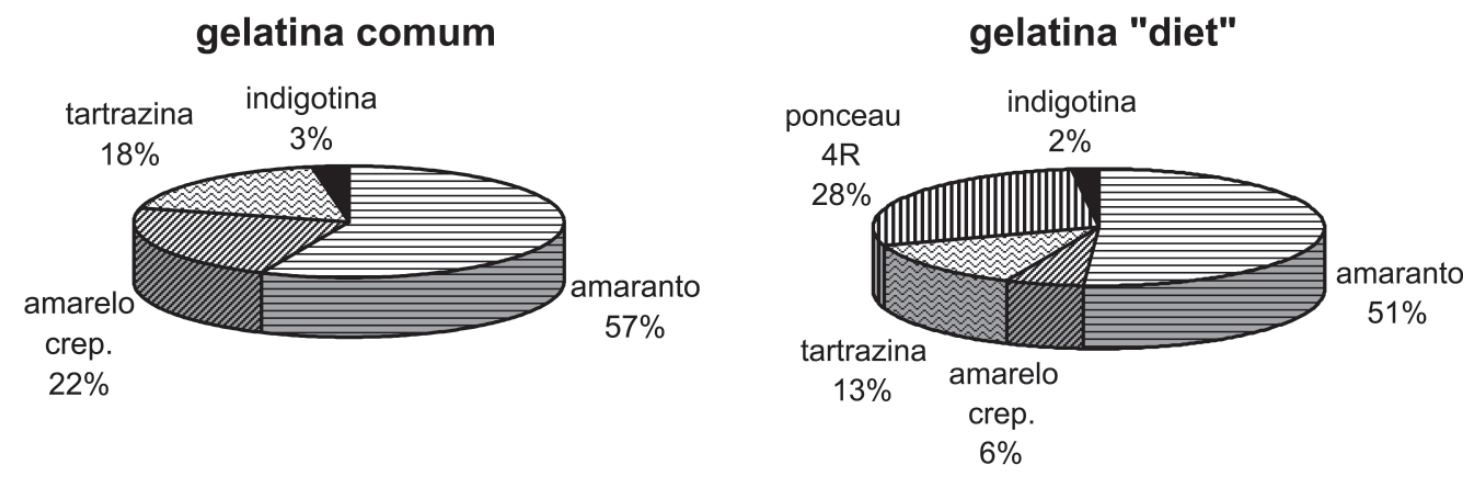

Figura 1. Gráficos das proporções dos corantes artificiais utilizados no preparo de pós para gelatinas 
Tabela 3. Comparação dos teores de corantes artificiais $(\mathrm{mg} / 100 \mathrm{~mL})$ entre lotes de pó para gelatina*

\begin{tabular}{|c|c|c|c|c|c|}
\hline Sabor & Corante & Marca & Lote 1 & Lote 2 & Lote 3 \\
\hline Morango & $\begin{array}{c}\text { tartrazina } \\
\text { amarelo crepúsculo } \\
\text { amaranto }\end{array}$ & $\begin{array}{l}\text { E } \\
\text { A } \\
\text { B } \\
\text { C } \\
\text { D } \\
\text { E } \\
\text { A } \\
\text { B } \\
\text { C } \\
\text { D } \\
\text { E }\end{array}$ & $\begin{array}{c}0,8 \pm 0,1 \mathrm{a} \\
3,4 \pm 0,2 \mathrm{a} \\
2,7 \pm 0,0 \mathrm{a} \\
3,5 \pm 0,5 \mathrm{a} \\
2,25 \pm 0,04 \mathrm{a} \\
1,6 \pm 0,2 \mathrm{a} \\
4,2 \pm 0,2 \mathrm{a} \\
3,7 \pm 0,3 \mathrm{a} \\
1,62 \pm 0,02 \mathrm{a} \\
1,40 \pm 0,06 \mathrm{a} \\
4,14 \pm 0,07 \mathrm{a} \\
\end{array}$ & $\begin{array}{c}0,75 \pm 0,02 \mathrm{a} \\
3,39 \pm 0,07 \mathrm{a} \\
3,1 \pm 0,2 \mathrm{~b} \\
3,25 \pm 0,08 \mathrm{a} \\
3,3 \pm 0,3 \mathrm{~b} \\
2,18 \pm 0,03 \mathrm{~b} \\
4,2 \pm 0,3 \mathrm{a} \\
4,3 \pm 0,1 \mathrm{~b} \\
1,4 \pm 0,2 \mathrm{~b} \\
1,69 \pm 0,02 \mathrm{~b} \\
4,3 \pm 0,4 \mathrm{a} \\
\end{array}$ & $\begin{array}{c}0,89 \pm 0,00 \mathrm{a} \\
3,71 \pm 0,01 \mathrm{a} \\
3,0 \pm 0,2 \mathrm{~b} \\
3,2 \pm 0,2 \mathrm{a} \\
3,25 \pm 0,00 \mathrm{~b} \\
1,80 \pm 0,04 \mathrm{a} \\
3,83 \pm 0,04 \mathrm{a} \\
4,14 \pm 0,04 \mathrm{~b} \\
1,4 \pm 0,1 \mathrm{~b} \\
1,70 \pm 0,1 \mathrm{~b} \\
4,3 \pm 0,2 \mathrm{a} \\
\end{array}$ \\
\hline Abacaxi & tartrazina & $\begin{array}{l}\text { A } \\
\text { B } \\
\text { C } \\
\text { D } \\
\text { E } \\
\text { A } \\
\text { B } \\
\text { C } \\
\text { D } \\
\text { E }\end{array}$ & $\begin{array}{c}1,57+0,02 \mathrm{a} \\
2,3+0,3 \mathrm{a} \\
3,3+0,5 \mathrm{a} \\
2,9+0,3 \mathrm{a} \\
0,8+0,2 \mathrm{a} \\
0,53 \pm 0,03 \mathrm{a} \\
0,46 \pm 0,05 \mathrm{a} \\
0,3 \pm 0,1 \mathrm{a} \\
0,20 \pm 0,05 \mathrm{a} \\
0,12 \pm 0,08 \mathrm{a} \\
\end{array}$ & $\begin{array}{c}1,5 \pm 0,1 \mathrm{a} \\
2,2 \pm 0,4 \mathrm{a} \\
3,5 \pm 0,5 \mathrm{a} \\
2,6 \pm 0,1 \mathrm{a} \\
0,89 \pm 0,01 \mathrm{a} \\
0,59 \pm 0,02 \mathrm{a} \\
0,40 \pm 0,05 \mathrm{a} \\
0,10 \pm 0,05 \mathrm{~b} \\
0,19 \pm 0,05 \mathrm{a} \\
0,11 \pm 0,07 \mathrm{a} \\
\end{array}$ & $\begin{array}{c}1,4 \pm 0,1 \mathrm{a} \\
2,23 \pm 0,09 \mathrm{a} \\
3,2 \pm 0,1 \mathrm{a} \\
2,7 \pm 0,3 \mathrm{a} \\
0,7 \pm 0,2 \mathrm{a} \\
0,45 \pm 0,01 \mathrm{a} \\
0,41 \pm 0,07 \mathrm{a} \\
0,21 \pm 0,03 \mathrm{c} \\
0,23 \pm 0,00 \mathrm{a} \\
0,07 \pm 0,00 \mathrm{a} \\
\end{array}$ \\
\hline Limão & tartrazina & $\begin{array}{l}\text { A } \\
\text { B } \\
\text { C } \\
\text { D }\end{array}$ & $\begin{array}{l}1,61 \pm 0,04 \mathrm{a} \\
1,56 \pm 0,05 \mathrm{a} \\
2,05 \pm 0,00 \mathrm{a} \\
1,97 \pm 0,02 \mathrm{a} \\
\end{array}$ & $\begin{array}{c}1,63 \pm 0,01 \mathrm{a} \\
1,61 \pm 0,02 \mathrm{a} \\
1,6 \pm 0,1 \mathrm{~b} \\
1,7 \pm 0,1 \mathrm{a} \\
\end{array}$ & $\begin{array}{l}1,58 \pm 0,07 \mathrm{a} \\
1,65 \pm 0,00 \mathrm{a} \\
2,13 \pm 0,07 \mathrm{a} \\
1,53 \pm 0,03 \mathrm{~b} \\
\end{array}$ \\
\hline Cereja & azul de indigotina & $\begin{array}{l}\text { A } \\
\text { B } \\
\text { C } \\
\text { D } \\
\text { A } \\
\text { B } \\
\text { D } \\
\text { E }\end{array}$ & $\begin{array}{c}0,35 \pm 0,00 \mathrm{a} \\
0,60 \pm 0,02 \mathrm{a} \\
0,67 \pm 0,03 \mathrm{a} \\
0,20 \pm 0,01 \mathrm{a} \\
10,6 \pm 0,3 \mathrm{a} \\
6,6 \pm 0,5 \mathrm{a} \\
4,79 \pm 0,01 \mathrm{a} \\
3,0 \pm 0,2 \mathrm{a}\end{array}$ & $\begin{array}{c}0,41 \pm 0,02 \mathrm{a} \\
0,55 \pm 0,03 \mathrm{a} \\
0,5 \pm 0,2 \mathrm{a} \\
0,23 \pm 0,02 \mathrm{a} \\
10,4 \pm 0,7 \mathrm{a} \\
6,8 \pm 0,2 \mathrm{a} \\
5,1 \pm 0,2 \mathrm{a} \\
1,7 \pm 0,3 \mathrm{~b}\end{array}$ & $\begin{array}{c}0,72 \pm 0,03 \mathrm{~b} \\
1,05 \pm 0,02 \mathrm{~b} \\
0,21 \pm 0,03 \mathrm{~b} \\
0,60 \pm 0,00 \mathrm{~b} \\
9,9 \pm 0,3 \mathrm{a} \\
6,84 \pm 0,07 \mathrm{a} \\
6,2 \pm 0,4 \mathrm{~b} \\
2,8 \pm 0,2 \mathrm{a}\end{array}$ \\
\hline
\end{tabular}

*médias e estimativa de desvio padrão $(\mathrm{n}=4)$; letras iguais na mesma linha indicam que não há diferença significativa, nível de 5\% de significância

Tabela 4. Comparação dos teores de corantes artificiais $(\mathrm{mg} / 100 \mathrm{~mL})$ entre lotes de pó para gelatina*

\begin{tabular}{|c|c|c|c|c|c|}
\hline Sabor & Corante & Marca & Lote 1 & Lote 2 & Lote 3 \\
\hline Pêssego & $\begin{array}{c}\text { tartrazina } \\
\text { amarelo crepúsculo }\end{array}$ & $\begin{array}{l}\text { A } \\
\text { E } \\
\text { A } \\
\text { E }\end{array}$ & $\begin{array}{c}0,43 \pm 0,09 \mathrm{a} \\
1,05 \pm 0,09 \mathrm{a} \\
0,55 \pm 0,02 \mathrm{a} \\
0,8 \pm 0,4 \mathrm{a} \\
\end{array}$ & $\begin{array}{c}0,39 \pm 0,04 \mathrm{a} \\
1,1 \pm 0,2 \mathrm{a} \\
0,39 \pm 0,04 \mathrm{~b} \\
0,07 \pm 0,00 \mathrm{a} \\
\end{array}$ & $\begin{array}{l}0,38 \pm 0,06 \mathrm{a} \\
2,28 \pm 0,01 \mathrm{~b} \\
0,38 \pm 0,06 \mathrm{~b} \\
0,12 \pm 0,00 \mathrm{a} \\
\end{array}$ \\
\hline$\overline{\text { Uva }}$ & $\begin{array}{l}\text { amaranto } \\
\text { azul de indigotina }\end{array}$ & $\begin{array}{l}\text { A } \\
\text { B } \\
\text { C } \\
\text { D } \\
\text { A } \\
\text { B } \\
\text { C } \\
\text { D } \\
\end{array}$ & $\begin{array}{c}3,2 \pm 0,4 \mathrm{a} \\
3,10 \pm 0,02 \mathrm{a} \\
3,46 \pm 0,07 \mathrm{a} \\
2,49 \pm 0,07 \mathrm{a} \\
0,64 \pm 0,08 \mathrm{a} \\
0,49 \pm 0,05 \mathrm{a} \\
0,46 \pm 0,09 \mathrm{a} \\
0,22 \pm 0,04 \mathrm{a} \\
\end{array}$ & $\begin{array}{c}2,4 \pm 0,4 \mathrm{~b} \\
2,19 \pm 0,07 \mathrm{~b} \\
3,5 \pm 0,7 \mathrm{a} \\
3,4 \pm 0,2 \mathrm{~b} \\
0,46 \pm 0,04 \mathrm{~b} \\
0,54 \pm 0,07 \mathrm{a} \\
0,17 \pm 0,03 \mathrm{~b} \\
0,27 \pm 0,02 \mathrm{a} \\
\end{array}$ & $\begin{array}{c}2,14 \pm 0,05 \mathrm{~b} \\
3,79 \pm 0,01 \mathrm{c} \\
3,0 \pm 0,1 \mathrm{~b} \\
2,4 \pm 0,2 \mathrm{a} \\
0,71 \pm 0,07 \mathrm{a} \\
0,72 \pm 0,02 \mathrm{~b} \\
0,22 \pm 0,00 \mathrm{~b} \\
0,21 \pm 0,02 \mathrm{a} \\
\end{array}$ \\
\hline Tutti-Frutti & $\begin{array}{c}\text { tartrazina } \\
\text { amarelo crepúsculo } \\
\text { amaranto }\end{array}$ & $\begin{array}{l}A \\
E \\
A \\
E \\
A \\
E\end{array}$ & $\begin{array}{c}1,08 \pm 0,02 \mathrm{a} \\
2,93 \pm 0,01 \mathrm{a} \\
2,13 \pm 0,05 \mathrm{a} \\
0,85 \pm 0,07 \mathrm{a} \\
1,31 \pm 0,07 \mathrm{a} \\
4,2 \pm 0,3 \mathrm{a}\end{array}$ & $\begin{array}{c}1,07 \pm 0,06 \mathrm{a} \\
3,17 \pm 0,09 \mathrm{a} \\
2,20 * 0,04 \mathrm{a} \\
0,9 \pm 0,1 \mathrm{a} \\
1,4 \pm 0,2 \mathrm{a} \\
4,9 \pm 0,3 \mathrm{a}\end{array}$ & $\begin{array}{c}0,8 \pm 0,3 \mathrm{~b} \\
3,2 \pm 0,3 \mathrm{a} \\
1,9 \pm 0,7 \mathrm{a} \\
0,79 \pm 0,02 \mathrm{a} \\
0,9 \pm 0,2 \mathrm{~b} \\
4,7 \pm 0,7 \mathrm{a} \\
\end{array}$ \\
\hline Framboesa & $\begin{array}{c}\text { tartrazina } \\
\text { amarelo crepúsculo } \\
\text { amaranto }\end{array}$ & $\begin{array}{l}\text { E } \\
\text { A } \\
\text { D } \\
\text { A } \\
\text { D } \\
\text { E }\end{array}$ & $\begin{array}{c}0,9 \pm 0,2 \mathrm{a} \\
1,03 \pm 0,01 \mathrm{a} \\
1,5 \pm 0,2 \mathrm{a} \\
3,0 \pm 0,2 \mathrm{a} \\
2,48 \pm 0,04 \mathrm{a} \\
3,19 \pm 0,03 \mathrm{a} \\
\end{array}$ & $\begin{array}{c}0,84 \pm 0,04 \mathrm{a} \\
1,4 \pm 0,4 \mathrm{~b} \\
1,6 \pm 0,2 \mathrm{a} \\
3,15 \pm 0,08 \mathrm{a} \\
3,40 \pm 0,03 \mathrm{~b} \\
3,19 \pm 0,03 \mathrm{a} \\
\end{array}$ & $\begin{array}{c}0,41 \pm 0,02 \mathrm{~b} \\
1,46 \pm 0,02 \mathrm{~b} \\
1,4 \pm 0,3 \mathrm{a} \\
2,85 \pm 0,01 \mathrm{a} \\
2,73 \pm 0,03 \mathrm{c} \\
3,3 \pm 0,2 \mathrm{a} \\
\end{array}$ \\
\hline Tangerina & $\begin{array}{c}\text { tartrazina } \\
\text { amarelo crepúsculo }\end{array}$ & $\begin{array}{l}\text { A } \\
\text { D } \\
\text { A } \\
\text { D }\end{array}$ & $\begin{array}{c}0,50 \pm 0,02 \mathrm{a} \\
0,14 \pm 0,00 \mathrm{a} \\
1,1 \pm 0,2 \mathrm{a} \\
0,5 \pm 0,3 \mathrm{a} \\
\end{array}$ & $\begin{array}{l}0,47 \pm 0,06 \mathrm{a} \\
0,17 \pm 0,04 \mathrm{a} \\
1,27 \pm 0,07 \mathrm{~b} \\
0,43 \pm 0,01 \mathrm{a} \\
\end{array}$ & $\begin{array}{c}0,42 \pm 0,07 \mathrm{a} \\
0,18+0,03 \mathrm{a} \\
1,31+ \pm 0,01 \mathrm{~b} \\
0,4 \pm 0,1 \mathrm{a} \\
\end{array}$ \\
\hline
\end{tabular}

\footnotetext{
*médias e estimativa de desvio padrão $(n=4)$; letras iguais na mesma linha indicam que não há diferença significativa, nível de 5\% de significância
} 
a)

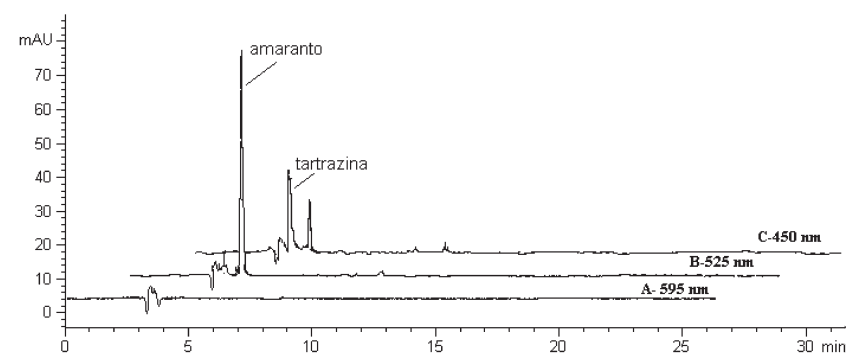

b)

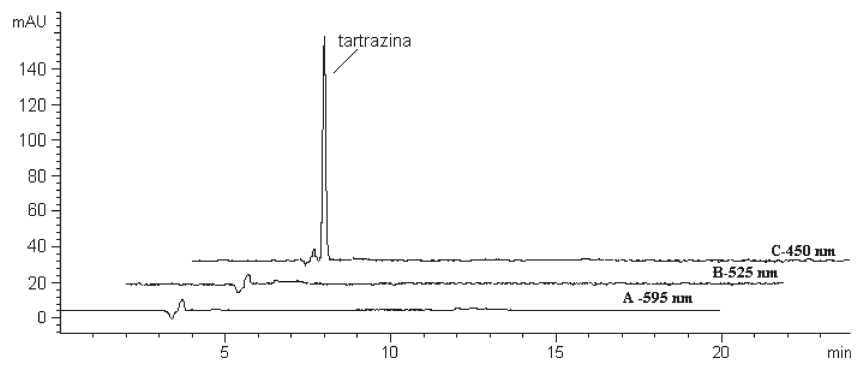

Figura 2. Perfis cromatográficos nos três comprimentos de onda de leitura para: (a) gelatina comum sabor framboesa; (b) gelatina "diet" sabor abacaxi. Condições cromatográficas descritas no texto

\section{CONCLUSÕES}

Na maioria das amostras de gelatina analisadas o limite estabelecido pela legislação brasileira não foi ultrapassado. Somente em duas amostras de gelatina sabor cereja, fabricante A, foram encontrados níveis um pouco superiores. Também não foram encontrados misturas de mais de três corantes e corantes não permitidos.

$\mathrm{O}$ amaranto foi o corante quantitativamente mais encontrado, estando presente em maior número de amostras. O ponceau $4 \mathrm{R}$ foi encontrado apenas nas amostras "diet".
Comparando-se os teores de corantes entre as gelatinas comum e "diet", observou-se uma tendência do uso de quantidades maiores em produtos "diet".

A composição qualitativa e quantitativa encontrada nos diferentes lotes para cada fabricante mostrou o bom controle na utilização dos corantes sintéticos.

Em vista dos resultados obtidos, a metodologia aqui aplicada mostrou-se bastante satisfatória, demonstrando um bom potencial para a sua aplicação em outros tipos de produtos alimentícios.

\section{REFERÊNCIAS}

1. Prado, M. A.; Godoy, H. T. ; J. Liq. Chromotogr. Relat. Technol. 2002, $25,2455$.

2. Riedel, G.; Controle Sanitário dos Alimentos, São Paulo: Loyola, 1987.

3. Clydesdale, F. M.; Crit. Rev. Food Sci. Nutr. 1993, 33, 83.

4. Sato, G. S.; Chabaribery, D.; Maia, M. L.; Carvalho, F. C.; Neto, A. N. Marques, S. A.; Agricultura em São Paulo 1992, 39 (supl. 1), 1.

5. http://www.anvisa.gov.br, acessada em Julho 2002.

6. Almeida, F. L.; I Simpósio sobre Aditivos para Alimentos, Campinas, SP, 1987.

7. Amaral, E. C. C.; Anais do seminário Latino Americano sobre Toxicologia de Alimentos, Campinas, SP, 1984.

8. Ozdemir, Y.; Akkan, A. A.; Turk. J. Chem. 1999, 23, 221

9. Chen, Q.; Mou, S.; Hou, X.; Riviello, J. M.; Ni, Z.; J. Chromatogr., A 1998, 827,73 .

10. Gennaro, M. C.; Gioannini, E.; Angelino, S.; Aigotti, R.; Giacosa, D.; J. Chromatogr., A 1997, 767, 87.

11. Gratzfeld-Hüsgen, A.; Schuster, R.; Catálogo Hewlett Packard, 1995, publication number 12-5964-3559E.

12. Greenway, G. M.; Kometa, N.; Macrae, R.; Anal. Methods Sect. 1992, 43, 137.

13. White, P. C.; Harbin A.; Analyst 1989, 114, 877.

14. Lancaster, F. E.; Lawrence, J. F.; J. AOAC 1983, 66, 1424.

15. Lancaster, F. E.; Lawrence, J. F.; J. AOAC 1982, 65, 1305.

16. Lawrence, J. F.; Lancaster, F. E.; Conacher, H. B. S.; J. Chromatogr. 1981, 210,168

17. McKone, H. T.; Ivie, K.; J. Chem. Educ. 1980, 57, 321.

18. Boley, N. P.; Bunton, N. G.; Crosby, N. T.; Johnson, A. E.; Roper, P.; Sommers, L.; Analyst 1980, 105, 589.

19. Puttermans, M. L.; Dryon, L.;Massart, D. L.; J. AOAC 1981, 64, 1.

20. Soares, L. M. V.; Rev. Inst. Adolf Lutz 2001, 60, 79.

21. Yanuka, Y.; Shalon, Y.; Weissenberg, E.; Nir-Grosfeld, I.; Analyst 1963, 88, 872. 